\title{
Customized Foot Orthosis Development by 3D Reconstruction of the CT Images
}

\author{
Sathish Kumar Paul ${ }^{1}$, Sudesh Sivarasu ${ }^{2}$, Lazar Mathew ${ }^{3}$ \\ ${ }^{1}$ Biomedical Engineering Division, VIT University, Vellore, India \\ ${ }^{2}$ Biomedical Engineering Division, Faculty of Health Science, University of Cape Town, Cape Town, South Africa \\ ${ }^{3}$ PSG Tech, Coimbatore, India \\ Email: sudeshsivarasu@gmail.com
}

Received July 20, 2012; revised August 22, 2012; accepted September 2, 2012

\begin{abstract}
Clinicians can provide a means to better distribute the pressure around the foot, and can also correct the biomechanics of the foot by using a customized shoe insole. If detected early enough, orthopedic insoles can correct or prevent further complications. In this study the 3 Dimensional (D) model of the foot was used to fabricate a customized orthosis. The Computed Tomography (CT) images of patient's foot having no muscle weakness and joint restriction were acquired. The gray intensities corresponding to the bones of the foot from the CT images were 3 dimensionally reconstructed. The 3D model of the foot was then imported into the CAD Software. Boolean operations were carried out in between the 3D foot model and a solid rectangular surface to create a customized foot orthosis. The exact contours and shape of the subject's foot was acquired using the computerized method of fabricating an orthosis. The novel idea described in this study support, automating the process of designing a customized orthosis with the impression got from the 3 dimensionally modeled feet, thereby reducing the modeling time considerably. The simple technique used in this process will help in giving comfort and stability to the patient's feet while walking.
\end{abstract}

Keywords: Customized Orthosis; 3 Dimensional Foot Model; Plantar Pressure

\section{Introduction}

The early prediction and management of plantar ulceration in patients with anesthetic foot such as in leprosy and diabetes have been major challenges in the field of health care all over the world. Sensory loss along with the underlying pathomechanics is one of the main causes for plantar ulcers in these patients. With increasing number of diabetic foot cases, the problem requires more surgical interventions. And the focus has largely shifted from the treatment of ulcers to the prevention of these ulcers.

$\mathrm{H}$. Cross in his study has demonstrated that the use of orthosis in the footwear could curtail the duration of ulcer than footwear without an orthosis [1]. The normal footwear used for the anesthetic feet does not accommodate the biomechanics of the foot [2]. A customized insole molded to the foot's shape at an optimal position will help preventing ulcers in anesthetic feet.

A properly fitted orthosis improves the joint functions of the hip, knee and the ankle other than improving the abnormal foot function, so it is necessary to take utmost care in the fabrication of the foot orthosis. The orthotic measurements are carried out either by the Anatomical Volumetric foot model (AVFM) or the Extrapolated Vo- lumetric foot model (EVFM) [3].

In the physical AVFM the impression of the foot geometry have not been reliable due to inherent problems in the measurement like the patient not being able to maintain the position or the therapist not able to hold the patient's foot in position. This process may fail sometimes due to the type of the material used for casting.

The cost involved in the Digital Volumetric Foot Model (DVFM) is high and are not preferred much by patients. The hand held scanners take much time to scan the foot and the patient is not able to maintain the position for the length of time.

The 3D modeling of the foot, by reconstructing the CT images has to a larger extent helped in the identification of the high tissue stress and in determining the peak plantar pressure of the foot. Patil et al. in his 2D model of the foot demonstrated that the shape of the foot and the muscle paralysis contribute to the stress changes in the different regions of the foot [4]. It has also been found that the non uniform variations in the soft tissue thickness of the sole lead to ulcer in the diabetic patients. [5] A novel 3D numerical model of the foot was developed incorporating all the geometrical and material properties of both skeletal and soft tissue components of 
the foot to find out the various stresses during different stance phases of gait [6]. This 3D model developed by Gefen et al., was used to help the surgeons and the therapists in their decision making skills by virtual removal of some bony and soft tissue elements until an optimal structure is obtained to enhance structural foot corrections in patients. This is obtained from work inspired from Sivarasu et al., 2007-2008 [7,8].

Many patients affected by Hansen's disease belong to the low socio economic group and are either not able to afford the cost associated with these assessment methods or these facilities are not able to reach them.

In this study the authors intend to create a customized functional foot orthosis without the casting, based on the simplified 3D model of the foot in the optimal position when the subtalar joint is in neutral position (neither pronated nor supinated).

\section{Methedology}

\subsection{D Modeling Methodology}

\subsubsection{Image Generation}

The CT scan was done on a patient affected by Hansen's disease. The CT images were obtained for the entire subject's keeping their foot in subtalar neutral position, the ankle in 90 degrees and in non weight bearing position.

\subsubsection{D Reconstruction}

The CT images acquired in the DICOM format for the $3 \mathrm{D}$ reconstruction are constituted by pixels with different gray intensities. The gray intensities corresponding to the bones and soft tissues of the foot were taken for the 3D reconstruction. The different phases of the 3D reconstruction using the MIMICS software are given below.

\subsubsection{Importing the Dicom Images}

Using the MIMICS software all the slices of the CT scan was imported. For more reproducibility of the CT data, the images were acquired with a slice distance of $1 \mathrm{~mm}$.

\subsubsection{Thresholding}

The thresholding based on the Hounsfield units were used to separate a bone from the other soft tissues like the muscles and the ligaments of the foot. In order to include both the cortical and the trabecular bones of the foot, the lower and the upper limit of the Hounsfield units were kept as $200 \mathrm{HU}$ to $2000 \mathrm{HU}$.

\subsubsection{Segmentation}

The segmentation operation was done on the multiple layer 3D slices. In this study geometrical separation of individual bone was not carried out in the foot and the segmentation process was made easier and can possibly be done by any clinician or therapist. Cavity fill opera-

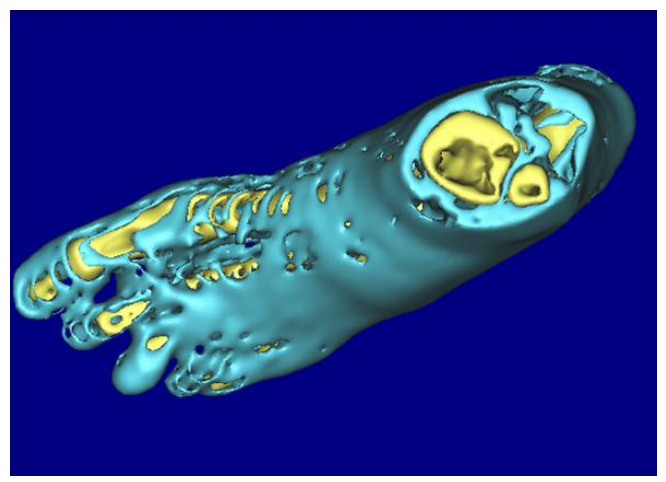

Figure 1. 3D model of the foot.

tions were done to remove voids in the model; polylines were also generated for this operation. Figure 1 shows the 3 dimensional models after segmentation.

\subsection{Modeling with AutoCAD}

\subsubsection{Importing the 3D Foot Model}

The 3D model of foot in the standard triangular language (stl) format was imported into the AutoCAD software. The model was then converted into a solid foot model using a mesh to solid converter.

\subsubsection{Creating a Rectangular Solid Model}

A rectangular base for the solid foot model was created in the AutoCAD software as per the dimensions of the plantar surface of the 3D foot model created. The rectangular base was then saved as a solid model. The Figure 2 shows the rectangular solid model created as per the dimensions of the 3D foot model.

\subsection{Boolean Operation}

The Boolean operation in the AutoCAD which is used to combine one or more primitives to form a common solid is also used to illustrate the relationship between the individual items that make up the solid model $[9,10]$.

Using Boolean operation the 3D foot model was combined with the solid rectangular model. Figure 3 shows the 3D foot model added with the rectangular base. The 3D foot model was then subtracted from the solid rectangular surface model. After the foot image was subtracted the impression of the foot was acquired in the solid rectangular model. The unwanted edges and surfaces of the rectangular model were edited and the shape that of the insole acquired. Filleting of the model was then done to get a smooth surface on the edges of the orthosis.

\subsection{Rapid Prototyping}

The orthotic model developed in the AutoCAD was fabricated by importing the (stl) images to the rapid proto 


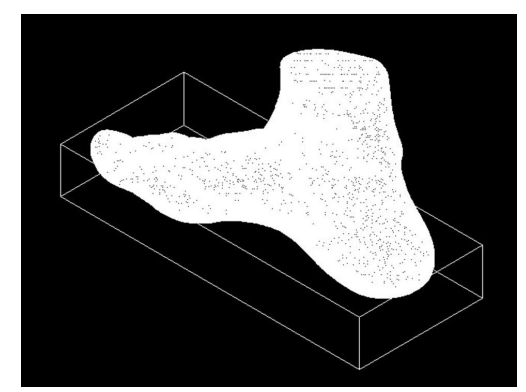

Figure 2. Solid foot model fitted with a rectangular solid base.

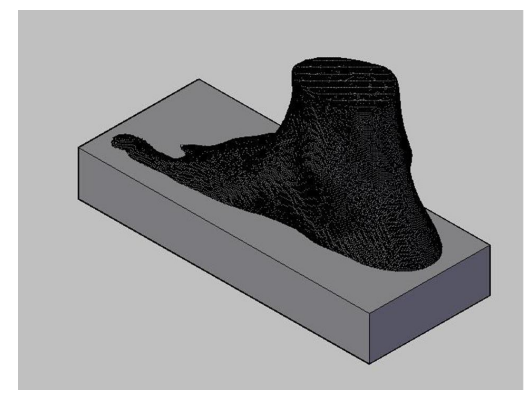

Figure 3. Solid foot model added with rectangular solid base.

typing machine. Quarter length insole of the foot orthosis was prototyped.

\section{Results}

The prototype developed from the images showed that the exact plantar contours of the foot can be acquired through this computerized foot orthotic development method. Figure 4 shows the impression of the orthosis designed in the AutoCAD and the Figure 5 shows the prototype of the customized orthosis fabricated. The quarter length orthosis was then fitted on the patient's feet. The impression of the orthosis was accurate in fitting to the contours of the plantar aspect of the patient's feet.

\section{Discussion}

The results demonstrate that the computerized orthotic fabrication method followed in this study was found to be more reliable in acquiring the anatomical contours of the plantar foot surface for orthotic fabrication. The cost involved in the material used for the moulding processes like the Plaster of Paris Powder (POP), POP rolls and the wastage present in the manual method when we use materials like Ethyl Vinyl Acetate (EVA) and the thermoplastics are reduced by this method. Some of the other advantages of this method of orthotic fabrication include:

\subsection{Easy Storage and Transfer of Data}

All the details of the CT images are stored in DICOM image format and the foot model that are reconstructed

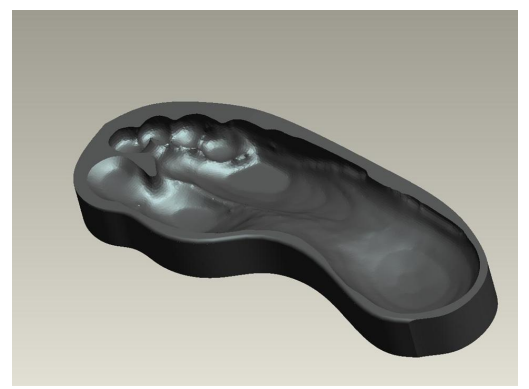

Figure 4. Full length customized orthosis designed.

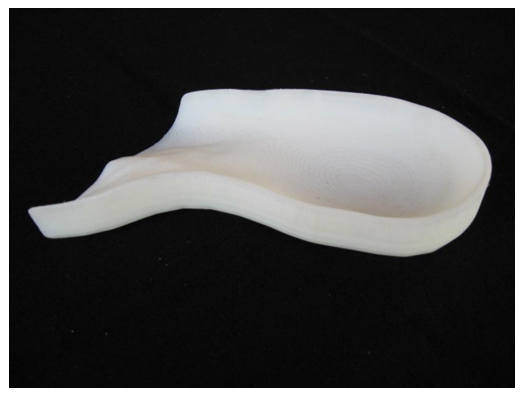

Figure 5. Prototype of customized orthosis fabricated.

from the CT images are stored in STL, ACIS or the IGES format. These formats can be easily transferred to different places through the available technology. This helps in the people from the remote parts of a country to be able to access the advanced medical care.

\subsection{Simple Design Technique}

Simple techniques are used in the fabrication of this orthosis. This will help in the medical staff like the Doctors, Therapists and the Podiatrists having less engineering skills to fabricate an orthosis. The need for operating the software is enough to fabricate an orthosis.

\subsection{Comfort and Stability for the Patients}

This orthosis is customized to individual patient's feet. This will help in the comfort of the patient when he uses the orthosis in the footwear while walking.

This orthosis fabrication method takes into account all the bony prominences and the anatomical contours of the part of the body. This will give more stability to the part of the patient's body where the orthosis is fixed.

\subsection{Bulk Manufacturing}

The orthosis can be manufactured in bulk if necessary and it will help in the reduction of the manufacturing cost. The library of the impression data will help in the retrieval of patient details.

\section{Conclusion}

Orthopedic foot wear plays an important role in the treat- 
ment and the prevention of ulcer in the diabetic as well as the leprosy patients. The current work is a novel technique in the fabrication of orthosis. This method reduces the use of more cumbersome techniques to capture the foot images and to take the cast measurements. The simple method used in this study to fabricate a customized orthosis will help in reducing the plantar ulcer and its consequences considerably in a cost efficient way.

\section{REFERENCES}

[1] H. Cross, V. N. Kulkarni, A. Dey and G. Rendall, "Plantar Ulceration in Patients with Leprosy," Journal of Wound Care, Vol. 5, No. 9, 1996, pp. 406-411.

[2] R. L. Valmassay, "Clinical Biomechanics of the Lower Extremities,” Mosby, Maryland Heights, 1996.

[3] PFOLA Technical Standards Document, "Foot Orthotic Classification, Definitions, and Summary of Manufacturing processes document," 2012.

http://www.nvo.com/pfola/nss-folder/publicfolder/Micros oft\%20Word\%20-\%20PFOLA\%20Technical\%20Standar ds\%20Document\%2011.03.09.pdf

[4] V. J. Thomas, K. M. Patil, S. Radhakrishnan, et al., "Three Dimensional Stress Analysis for the Mechanics of
Plantar Ulcers in Diabetic Neuropathy,” Medical and Biological Engineering and Computing, Vol. 42, No. 2, 2004, pp. 230-235. doi:10.1007/BF02344636

[5] E. T. Sagrlatto, “A Compendium of Podiatric Biomechanics,” California College of Podiatric Medicine Corporation, San Fransisco, 1971.

[6] A. Gefen, M. Megido-Ravid, Y. Itzchak and M. Arcan, "Biomechanical Analysis of the Three Dimensional Foot Structure during Gait: A Basic Tool for Clinical Applications,” Journal of Biomechanical Engineering, Vol. 122, No. 6, 2000, pp. 630-639. doi:10.1115/1.1318904

[7] S. Sivarasu and L. Mathew, "Finite-Element-Based Design Optimisation of a Novel High Flexion Knee Used in Total Knee Arthroplasty,” Applied Bionics and Biomechanics, Vol. 5, No. 2, 2008, pp. 77-87. doi:10.1080/11762320802564200

[8] S. Sivarasu and L. Mathew, “3D CAD Conceptual Design of an Artificial Knee," Journal of Long-Term Effects of Medical Implants, Vol. 17, No. 4, 2007, pp. 313-320. doi:10.1615/JLongTermEffMedImplants.v17.i4.50

[9] Z. Ibrahim, "Mastering CAD/CAM," McGraw Hill, New York, 2004, pp. 392-393.

[10] T. Sham, “AutoCAD 2007 for Engineers \& Designers," Dreamtech Press, New Delhi, 2006. 